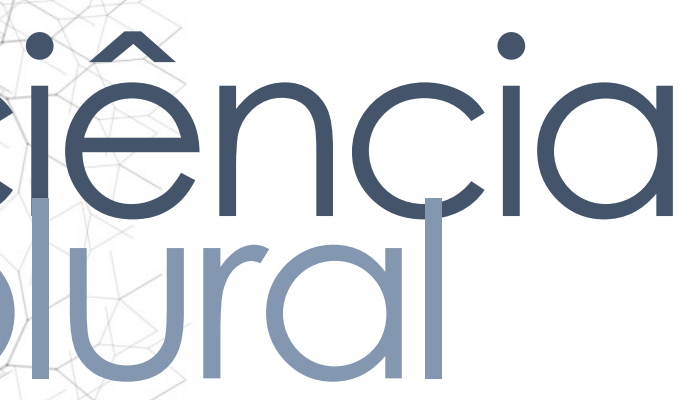

\title{
REPRESENTAÇÕES SOCIAIS DE ENFERMEIRAS DA ATENÇÃO BÁSICA SOBRE O PARTO NORMAL
}

\section{Social Representations of nurses of basic attention on normal birth}

Nayale Lucinda Andrade Albuquerque || Centro Universitário Tabosa de Almeida ASCES-UNITA || Doutoranda em Saúde Integral pelo Instituto de Medicina Integral Professor Fernando Figueira - IMIP || nayalealbuquerque@asces.edu.br

Eline Ferreira Mendonça || Centro Universitário Tabosa de Almeida ASCESUNITA | | Mestre em Avaliação em Saúde pelo Instituto de Medicina Integral Prof. Fernando Figueira (IMIP) | | elinemendonca@asces.edu.br

Marilia Cruz Gouveia Câmara Guerra || Centro Universitário Tabosa de Almeida ASCES-UNITA || Mestre em Enfermagem pela Universidade Federal de Pernambuco (UFPE) | | mariliacamara@asces.edu.br

Júlio César Bernardino da Silva || Centro Universitário Tabosa de Almeida ASCES-UNITA || Discente de Bacharelado em Enfermagem pelo Centro Universitário Tabosa de Almeida ASCES-UNITA cesarsilva04@hotmail.com

Helena Natalya da Silva Lins || Centro Universitário Tabosa de Almeida ASCES-UNITA || Enfermeira pelo Centro Universitário Tabosa de Almeida ASCES-UNITA | | 2013206019@app.asces.edu.br

Autor responsável pela correspondência

Nayale Lucinda Andrade Albuquerque | | nayalealbuquerque@asces.edu.br 


\section{RESUMO}

Introdução: A assistência desumanizada no parto pode originar informações negativas que são transmitidas entre as gerações, acarretando em representações sociais. Dessa forma, representações sociais do parto normal por parte do profissional enfermeiro, um dos protagonistas da assistência à saúde da mulher na atenção básica, são importante objeto de estudo, a partir do entendimento que estes profissionais constroem nas realidades em que vivem. Objetivo: Identificar as representações sociais de enfermeiras da atenção básica sobre parto normal. Método: Estudo descritivo, exploratório, qualitativo, realizado com 12 enfermeiras, responsáveis por Equipes de Saúde da Família e que tinham mais de 01 ano de experiência na atenção básica no município de Caruaru/PE, nordeste do Brasil. Foram realizadas entrevistas semi-estruturadas, guiadas pela questão norteadora: Fale-me sobre parto normal. As falas foram submetidas à análise de conteúdo temática proposta por Bardin e interpretadas à luz dos constructos da Teoria das Representações Sociais. Resultados: A partir da análise das falas, emergiram quatro categorias temáticas: Lei da vida, Desejo e negação do parto normal, Insegurança, Imposição para mulheres de baixa renda. Conclusões: As representações que emergiram das falas das enfermeiras apresentam o parto normal como um evento natural, permeado pelo auxílio divino, trazendo o desejo em vivenciá-lo. Entretanto, este fato não é suficiente para que haja uma escolha por este tipo de parto, devido a outros fatores, como a insegurança presente em diferentes momentos e aspectos, bem como a compreensão de que o parto normal é imposto nos serviços públicos de saúde. Dessa forma, compreender as representações sociais do parto normal, pela perspectiva da enfermagem atuante na atenção básica, propicia um olhar sob a educação em saúde no pré-natal, onde são reelaborados os conhecimentos que envolvem este evento.

Palavras-Chave: Parto Normal; Enfermagem; Atenção Primária à Saúde; Educação em Saúde 


\section{ABSTRACT}

Introduction: Dehumanized care at birth may give rise to negative information that is transmitted between generations, giving rise to social representations. Thus, these social representations of normal childbirth by the nurse practitioner, one of the protagonists of the assistance to women's health in basic care, are an important object of study, based on the understanding that these professionals construct in the realities in which they live. Objective: To identify the social representations of primary care nurses on normal delivery. Methods: A descriptive, exploratory, qualitative study was carried out with 12 nurses, who were responsible for Family Health Teams and who had more than 01 years of experience in basic care in the city of Caruaru / PE, northeastern Brazil. Semistructured interviews were conducted, guided by the guiding question: Tell me about normal delivery. The speeches were submitted to the analysis of thematic content proposed by Bardin and interpreted in the light of the constructs of Theory of Social Representations. Results: Based on the analysis of the speeches, four thematic categories emerged: Law of life, Desire and denial of normal birth, Insecurity, Imposition for low income women. Conclusions: The representations that emerged from the nurses' statements present normal childbirth as a natural event, permeated by divine help, bringing the desire to experience it. However, this fact is not enough to make a choice for this type of delivery, due to other factors such as the insecurity present at different times and aspects, as well as the understanding that normal delivery is imposed in public health services. In this way, understanding the social representations of normal childbirth, from the perspective of nursing acting in basic care, provides a perspective on prenatal health education, where the knowledge that involves this event is re-elaborated.

Keywords: Natural Childbirth; Nursing; Primary Health Care; Health Education 


\section{Introdução}

O cenário mundial apresenta um crescente aumento no número de partos cesarianos, com consequências para a morbimortalidade materno infantil ${ }^{1}$. A possibilidade de reduzir o sofrimento do parto pode levar as mulheres a escolherem a cesariana, mesmo quando os estudos mostram uma maior preferência pelo parto normal ${ }^{2,3}$. Uma assistência desumanizada no parto pode originar informações negativas que são transmitidas entre as gerações, originando representações sociais ${ }^{4,5}$.

Compreende-se que todo o processo educativo envolve o ser educador e o ser educado numa "troca de saberes" transformando este momento em um processo construído pelos dois atores ${ }^{6}$. Portanto, o profissional de saúde é considerado "um agente potencial de mudança" que, a partir de ações educativas, possibilitam discussões sobre "o senso comum e a ciência" 5,7.

Este processo gera a elaboração de teorias do senso comum, construindo uma rede de significações acerca de parto, de acordo com as classificações préexistentes de ambos os sujeitos, levando às escolhas das gestantes por determinados posicionamentos diante do parto normal ${ }^{5,8}$.

As representações sociais do parto normal do profissional enfermeiro, um dos protagonistas da assistência à saúde da mulher na atenção básica, são importante objeto de estudo, a partir do entendimento que estes profissionais constroem conhecimentos nas realidades em que vivem. A identificação das representações sociais das enfermeiras da atenção básica acerca do parto normal pode colaborar na compreensão de sua prática profissional, visualizando as possibilidades de promoção da educação para o parto. Portanto, o objetivo desta esquisa foi identificar as representações sociais de enfermeiras da atenção básica bre parto normal. 


\section{Método}

Trata-se de um estudo descritivo, exploratório, de abordagem qualitativa, embasado na Teoria das Representações Sociais (TRS). Esta teoria remete-se ao conhecimento do senso comum que se encontra compartilhado, servindo de referencial a respeito de um determinado objeto. Estudar o senso comum permite compreender as práticas sociais, justificando determinadas atitudes e comportamentos, explicando a realidade social ${ }^{5,9}$.

O estudo foi realizado com as enfermeiras das estratégias de saúde da família do município de Caruaru-PE. Entrevistou-se 12 enfermeiras com tempo de serviço de 01 ano ou mais, em unidades de saúde, no período de setembro de 2012 a janeiro de 2013. O número de participantes foi determinado pelo critério de saturação da amostra.

Para a coleta de dados, utilizou-se a entrevista semi-estruturada, gravada e orientada por um roteiro temático, após aceitação e assinatura do Termo de Consentimento Livre e Esclarecido. As entrevistas foram realizadas no próprio local de trabalho, em sala que permitisse conforto e privacidade às participantes. As gravações foram transcritas de forma integral, sendo todas as entrevistas submetidas à técnica de análise de conteúdo, na categoria temática ${ }^{10}$.

Realizar uma análise de conteúdo temática consiste em descobrir os núcleos de sentido, a partir da aplicação da grelha. As fases da Análise de Conteúdo constituem - se de: Pré - análise; Exploração do material; Tratamento dos resultados; Inferência e Interpretação. As falas foram transcritas na grelha de análise, procedendo-se a leitura flutuante das transcrições a fim de apreender o sentido global. Após este procedimento, uma leitura exaustiva das falas foi realizada para possibilitar a categorização, por meio da extração dos núcleos de entido encontrados e destacados nas falas, sem perder de vista o contexto, btendo-se os trechos das entrevistas que respondiam à questão ${ }^{10}$. 
A partir daí, as inferências foram realizadas categorizando os núcleos de sentido, inicialmente, em códigos (Confronto I) e depois em subcategorias (Confronto II) até obter as categorias temáticas (Confronto Final) ${ }^{10}$.

Quadro 1. Fases da Análise de Conteúdo Temática de Bardin.

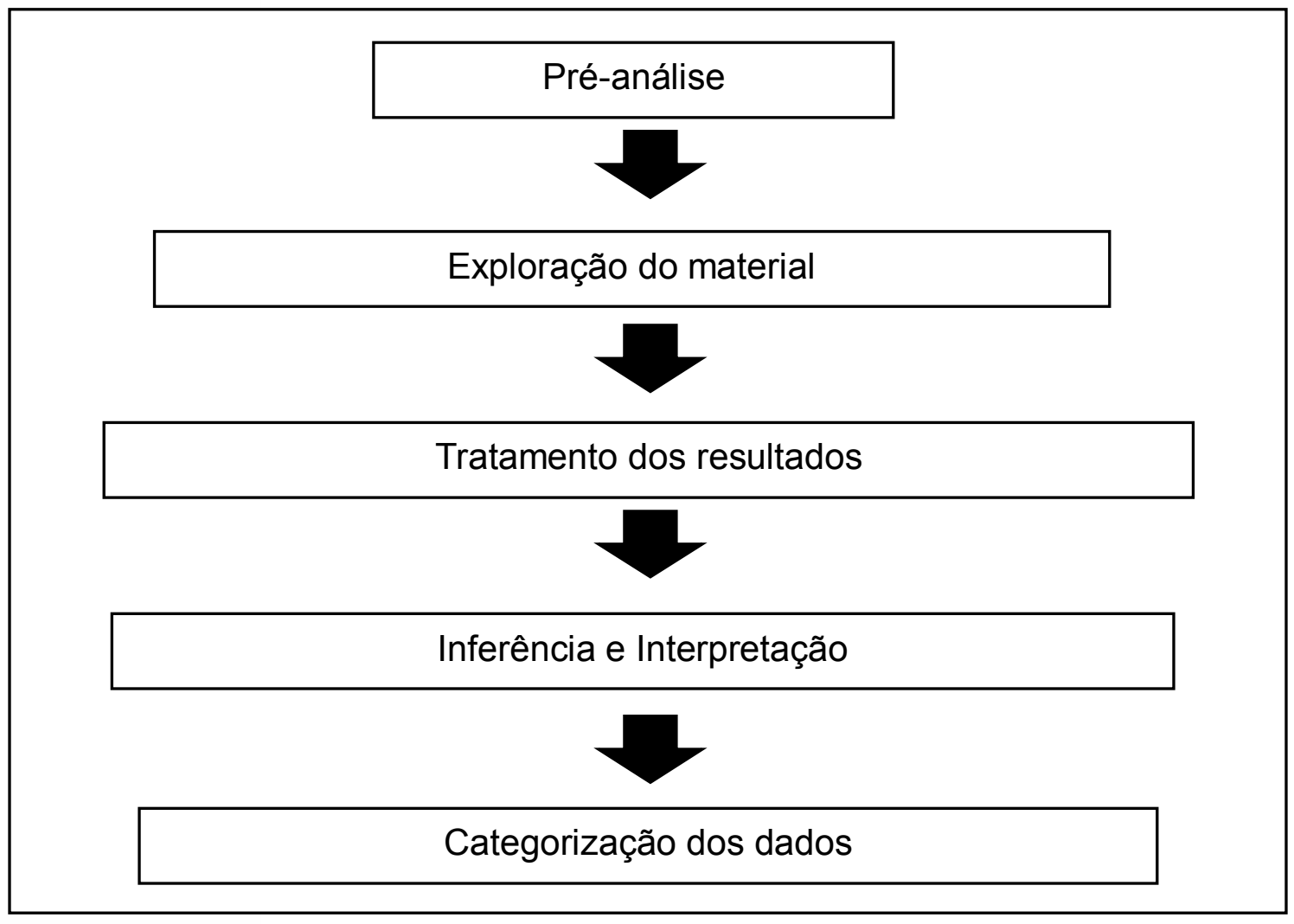

O estudo obteve a anuência do município para a sua realização e foi aprovado pelo Comitê de Ética em Pesquisa da Universidade Federal de Pernambuco, sob o protocolo no 94 806, de acordo com os requisitos da Resolução n 466 de 2012 do Conselho Nacional de Saúde (CNS), que trata das diretrizes e normas de pesquisa envolvendo seres humanos.

\section{Resultados e Discussão}

As enfermeiras possuíam idade entre 25 e 57 anos, sendo (58,33\%) delas com 1 a 3 anos de trabalho em estratégia de saúde da família (ESF), (8,33\%) com experiência de 06 anos e (33,33\%) com experiência de 11 a 16 anos de trabalho. Dentre as especialidades, $(16,66 \%)$ eram especialistas em saúde da mulher e 


\section{ciência
púral}

$(8,33 \%)$ estava com esta especialização em andamento. Quando à paridade, $(66,66 \%)$ das enfermeiras possuíam filhos, sendo verificado que $(41,66 \%)$ vivenciaram o parto cesariano, (8,33\%) vivenciou o parto normal, $(16,66 \%)$ experienciaram os dois tipos de parto e (33,33\%) eram nulíparas.

Após a análise temática das doze entrevistas, emergiram as seguintes categorias: Lei da vida; Desejo e negação do parto normal; Insegurança; Imposição para mulheres de baixa renda.

\section{Lei da Vida}

As falas revelaram uma representação do parto normal como um momento divino que gera força e superação no período expulsivo. É um momento repleto de religiosidade, existindo a crença de que uma força superior auxilia a mulher que o vivencia ${ }^{4}$.

... a gente encontra uma força sobrenatural, uma força que vem de Deus ... aquela força que a gente consegue na hora ... botar aquele filho pra fora ... Deus dá aquela força a gente. (E3)

As enfermeiras ainda referem ser, este tipo de parto, um momento natural onde a mulher nasceu pronta para esta missão. Esta representação é compartilhada nas comunicações sociais e são repassadas como herança social ${ }^{5,11}$.

... eu imagino que é assim, a naturalidade é a ordem natural das coisas. (E1)

\section{Desejo e negação do parto normal}

O desejo pelo parto normal foi relatado como um sonho regado pelas informações resgatadas no senso comum. Além desta representação, esse parto significa a vivência plena da sua feminilidade $\mathrm{e}^{11,12}$.

... meu sonho era que fosse parto normal ... desejo de ter o filho normal. (E11) 
... você se sente realizada, você se sente mulher, você se sente mãe, vem todos aqueles sentimentos sobre a sua mãe ... e esse sentimento é quando você se sente realizada, completada, não é... é quando você tem seu filho. A sensação de ter normal é isso aí. (E9)

Convém ressaltar a ligação deste desejo com os benefícios do parto normal, sempre lembrados pelas entrevistadas, emergindo uma representação deste parto como prática saudável ${ }^{3}$.

... você se sente bem no pós-parto. (E2)

... a recuperação pra mulher é bem mais rápida. (E4)

Em contrapartida, um dos relatos apresentou a negação do mesmo. Esta contradição é visualizada ao observar o elevado índice de partos cesarianos, apesar da preferência pelo parto normal ${ }^{1,3}$.

... Eu não faria... eu não faria. (E7)

A compreensão da negação do parto normal por gestantes atendidas pelas enfermeiras também foi citada, permitindo visualizar a influência dos conhecimentos partilhados no senso comum nas classificações dadas ao objeto em foco: o parto normal ${ }^{8}$.

... nenhuma mulher quando ela engravida a última coisa que ela quer é um parto normal. (E10)

A contradição entre o que se pensa e o que se faz, revela que o comportamento é determinado por múltiplos fatores imbricados na realidade social, gerando condutas 5 . Compreender as representações do parto normal permite analisar como este pensamento é construído, levando a atitudes que eralmente são influenciáveis no meio social ${ }^{8}$. 
Um sentimento ambíguo de desejo e receio diante de uma escolha pelo parto normal também é revelado nas falas quando as mesmas se perguntam o "por que não querer um parto normal".

... se as nossas mães, avós, bisavós, tataravós, tiveram parto normal, por que não a gente ter? ... se eu tenho condição de dar à luz por parto normal, por que não? (E5)

O sofrimento e a dor do parto são representações interligadas ao parto normal, contribuindo na epidemiologia obstétrica da atualidade. A imagem do parto normal à dor e sofrimento, é apresentada em alguns estudos qualitativos 12-14 e a origem destes sentimentos está ancorada nas informações lançadas por amigos, parentes sobre as experiências traumáticas com este tipo de parto ${ }^{4,12}$. Acrescenta-se que as informações da mídia consolidam este imaginário ${ }^{12}$.Assim, o entendimento destas representações auxilia no esclarecimento de como o ser humano ver o objeto social e como ele se relaciona com ela ${ }^{5}$.

... foi uma tortura o parto normal ... senti dor, senti que é uma coisa que... pra mim não é considerado normal, pra mim não era normal. (E3)

... sofrimento que sente, só isso. (E4)

\section{Insegurança}

A insegurança é uma representação social fortemente visualizada nas falas das enfermeiras, em diferentes contextos. Uma das formas de insegurança é observada a partir do sentimento de medo do parto normal, por vezes originado pelas comunicações estabelecidas com as gestantes atendidas. Neste sentido, as representações não são originadas individualmente, mas produzidas nas nterações sociais, a partir das exposições de ideias, crenças e imagens. Essa propriação da realidade permite o confronto com informações preexistentes do 
sujeito e uma reconstrução do objeto, sendo, o sujeito, um construtor ativo da realidade ${ }^{5}$.

... meio assustador né, parto normal é assustador ... eu vejo assim como profissional, as angústias quando elas (gestantes atendidas) vêm ... eu tinha muito medo de ter normal, medo da dor. (E10)

A representação de insegurança também é verificada quando as enfermeiras associam o parto normal a um risco potencial para complicações, a partir dos relatos que trazem a necessidade de uma estrutura assistencial hospitalar intensiva.

... a maternidade que eu fiz não tinha UTI neonatal, eu fiquei com medo, a minha insegurança era não ter UTI neonatal. (E2)

Outra enfermeira relata que a estrutura das salas de parto não é adequada para uma melhor assistência à mulher, havendo uma necessidade de readequação dos ambientes das salas de parto a fim de possibilitar privacidade à mulher e ações não farmacológicas de alívio da dor para sustentar as propostas de modificação desse cenário atual medicalizado ${ }^{15,16}$.

... qual é a unidade de saúde pública que tem uma triagem em uma maternidade onde se parteja com tranquilidade? Eu acho que nenhuma, né ... eu acho que nenhuma. (E6)

O médico também é gerador de uma representação do parto normal como prática insegura, quando sua opinião influencia o processo decisório das mulheres por um parto cesáreo ${ }^{17}$.

... nãa estão (os profissionais) tão adeptos (ao parto normal). Já que eles estão tão adeptos à cesariana, você fica com certo receio. (E12)

Algumas enfermeiras relatam os nascimentos em casa como uma opção reduz o sentimento de medo supracitado, diante do sentimento de 
acolhimento e familiaridade que este ambiente transmite. Conhecer o local do parto é importante, a fim de estabelecer um vínculo que auxilie na redução da insegurança.

... tinha uma parteira aqui, que fazia parto na casa dela, havia muito risco de infecção e tinha muita gente que preferia ter menino em casa. (E5)

... ficam meia que cortadas, não tem essa ligação entre esses dois tipos de serviço (primário e secundário). Aí isso gera insegurança, medo ... não tem um dia de visita na maternidade ... no público não funciona assim. Aí não tem essa integração. (E2)

Além deste vínculo, a presença de pessoas no apoio ao parto gera o sentimento de segurança. Uma revisão sistemática encontrou efeitos benéficos destes cuidadores, os quais promoveram redução do uso de medicamentos para dor, diminuição do número de cesarianas, discreta redução do tempo de parto ${ }^{18}$.

... esse obstetra me deixou insegura ... ele disse que tinha um congresso ... não ia poder ... (eu) correr o risco de ser com outro profissional ... infelizmente a gente não tem essa segurança (que tem no parto cesáreo) e o apoio desses profissionais nessa hora do parto. (E2)

... vai estar sozinha, né... vai estar sozinha ... é assustador ... vai estar sozinha, sem nenhum, sem companheiro perto, sem uma família. (E10)

... a gente tenta humanizar, trazer pra casa, trazer as doulas. (E6)

O preparo da mulher para o parto foi ressaltado pelas entrevistadas, orém visualizam-se nas consultas pré-natais mulheres geralmente tratadas mo coadjuvantes ${ }^{19}$. 
Um estudo de revisão não encontrou evidências científicas sobre a educação em saúde no pré-natal para o parto ${ }^{20}$. Porém, o processo educativo no pré-natal é estimulado, sendo apresentando uma medida essencial para a construção ativa da autonomia da mulher neste evento21.

Na educação em saúde no pré-natal, denota-se a mulher como ser social recebendo informações de variados campos de orientações, processando-as em seu universo interno. Estes dados são reconstituídos e lançados ao ambiente que a envolve, integrando suas concepções a uma rede de relações, que orientam comportamentos ${ }^{8}$.

... é um ambiente muito novo, então se ela, se a gente já for trabalhando isso durante o pré-natal com ela, ela vai chegar ali com menos angústias, mais segura. (E10)

... eu não tinha experiência, não tinha ... nunca fui orientada como era um parto normal, nem no meu pré-natal ... o médico nunca me orientou como era um parto normal, o que era que eu ia sentir, o que eu ia passar... não tinha essa orientação. (E3)

Outro fator promotor de insegurança é o desconhecimento da mulher em relação ao seu corpo biológico, quando ancoram o parto como um momento imprevisível, uma "caixinha de surpresas", podendo acarretar em danos maternos e neonatais ${ }^{22}$. Entretanto, há uma maior associação destes danos com o parto cesáreo 23 .

Diante deste fato, verifica-se que informações cristalizadas são repassadas entre os profissionais de saúde e continuam gerando condutas inadequadas. A constante atualização dos profissionais envolvidos na assistência à gestante e à parturiente é fundamental na recriação do senso comum 5 .

... o parto normal também pode trazer complicações e essas complicações podem surgir na hora. A gente não pode ter a 
garantia, não pode prever ... a criança nasceu com hipóxia e estava com circular de cordão, mas mesmo assim estava sendo insistido o trabalho de parto. (E4)

As falas das enfermeiras apontam a mídia e as orientações dos familiares como forma de preparo para o parto. Crenças e mitos sobre o parto normal baseados no modelo biomédico são apresentados às enfermeiras por várias fontes, como a mídia ${ }^{12}$. Estas informações apreendidas são repassadas nas suas condutas atuais, ancorando o parto normal em um momento onde a mulher é passiva.

... hoje as mulheres têm, hoje tem a experiência da familia ... você vê na televisão, tudo... elas tem orientação por todos os meios... fui preparada assim ... as pessoas mais antigas chegava pra gente $e$ dizia assim: olhe, não adianta gritar, você não vá gritando, porque ninguém vai the dar atenção, né ... Fui calma, segui todas as orientações dos profissionais no hospital. (E3)

Na hora, na sala, ela sempre seguir as orientações dos profissionais de saúde, que são ... eles estão ali capacitados, eles são treinados pra dar toda orientação a você, não adianta você chegar lá gritando. (E3)

A partir desse contexto, existe um reconhecimento quanto à necessidade de preparo dos próprios profissionais para a assistência ao parto. A formação acadêmica no Brasil é permeada por um modelo biomédico, culminando em insensibilidade e despreparo dos profissionais de saúde para o parto ${ }^{24}$. Esta condução é originada a partir dos conhecimentos compartilhados neste âmbito 5 .

... pra o parto normal tem que existir uma preparação. Tanto dos profissionais que estão orientando como da pessoa que está... da própria gestante que tem que se conscientizar com relação ao processo. (E12) 
... hoje em dia os profissionais obstetras, eles tão mais habilitados com o parto cesáreo do que o parto normal. (E2)

... são poucos obstetras que sabem partejar ... médicos não são formados pra isso (partejar). (E6)

Intervenções desnecessárias transmitidas nas salas de parto de forma rotineira estão presentes no imaginário das enfermeiras como uma forma de assistência desumana. Esta ideia gera insegurança, fazendo com que a mulher se perceba como um objeto passivo e indefeso nas mãos de quem detém o poder do saber ${ }^{5,19}$.

... você vai ser tocada o tempo inteiro ... é toque o tempo todo ... sem nenhuma educação (toque) ... o pessoal vai continuar parindo com o povo gritando no pé do ouvido ..."vamos, minha filha ... bote força", numa coisinha dura e fria feito essa mesa (bate na mesa) ... é assim que as pessoas vem ao mundo. (E6)

Eu vejo gestantes parturientes com laceração de colo, fazendo kristeller ... começa a fugir da normalidade, quando começa a se aplicar procedimentos ali, dentro do parto, que foge da normalidade, que fica assim querendo forçar aquele parto, então forçar de uma forma mecânica. (E12)

Neste momento, verifica-se a violência institucionalizada que percorre as maternidades em todo o mundo, com a ocorrência de maus tratos de diferentes naturezas que ferem a autonomia e a dignidade das mulheres ${ }^{15}$. Contudo, existe a possibilidade de mudança desta realidade a partir do entendimento de que o objeto é reconstruído em seu sistema de representações e a realidade é apreendida, passando de realidade objetiva para realidade subjetiva, ou seja, não produzindo a informação, mas reelaborando-a ${ }^{5,9}$.

posição às mulheres de baixa renda 
O parto normal também foi citado como uma imposição para a mulher pobre, perpetuando uma desigualdade de direitos sobre o corpo.

... no SUS é obrigada a fazer normal. (E2)

... na realidade a maioria deles eles vão ter o parto normal.

Estudos apontam que uma maior renda, maior escolaridade e mulheres da raça branca tem maior possibilidade de ter um parto cesariano, inclusive em maternidades públicas, sendo este parto culturalmente visto como um procedimento diferenciado ${ }^{2}$. Porém, no sistema público, onde existe um maior incentivo ao parto normal, o diálogo no pré-natal encontra-se ainda mais comprometido e muitas mulheres vivenciam o parto normal de uma forma passiva, aceitando todas as intervenções propostas e tidas como verdades absolutas pelas mesmas, culminando em experiências desagradáveis e até mesmo traumáticas. Este fato ocorre continuamente e é cristalizado por gerações $5,12,21$.

\section{Conclusões}

O parto normal é ancorado em relatos positivos de que é um evento natural, permeado pelo auxílio divino em sua evolução, trazendo o desejo em vivenciá-lo. Porém, também emergiram ancoragens deste evento na insegurança e numa imposição dos serviços públicos de saúde, fazendo com que este desejo não se concretize.

A partir da teia de significados, gerada nesse estudo, observa-se que as mulheres necessitam de uma rede de apoio ao parto normal que consiste em estrutura física adequada, recursos humanos qualificados e aplicação de uma assistência baseada em evidências científicas. Percebe-se ser imprescindível o colhimento da mulher, garantindo a manutenção da sua dignidade e o respeito seus direitos. 
Compreender as representações sociais do parto normal, pela perspectiva da enfermagem atuante na atenção básica, propicia um olhar sob a educação em saúde no pré-natal, onde são reelaborados os conhecimentos que envolvem este evento. Este olhar também aponta para a atenção à educação permanente destes profissionais, podendo favorecer a geração de representações sociais positivas do parto normal e auxiliar em condutas éticas e humanas às mulheres, contribuindo na desconstrução do modelo biomédico instituído na saúde pública vigente.

\section{Referências}

1. Lumbiganon P, Laopaiboon M, Gülmezoglu AM, Souza JP, Taneepanichskul $\mathrm{S}$, Ruyan P, et al. Method of delivery and pregnancy outcomes in Asia: the WHO global survey on maternal and perinatal health 2007-08. Lancet 2010 Feb 6.375(9713):490-9.

2. Mandarino NR, Chein MBC, Júnior FCM, Brito LMO, Lamy ZC, Nina VJS, et al. Aspectos relacionados à escolha do tipo de parto: um estudo comparativo entre uma maternidade pública e outra privada, em São Luís, Maranhão, Brasil. Cad. Saúde Pública. 2009 jul 25(7):1587-1596.

3. Stoll K, Fairbrother N, Carty E, Jordan N, Miceli C, Vostrcil Y, Willihnganz L, et al. "It's All the Rage These Days": University Students' Attitudes Toward Vaginal and Cesarean Birth. Birth. 2009 Jun 36(2):133-40.

4. Bezerra MGA, Cardoso MVLML. Fatores culturais que interferem nas experiências das mulheres durante o trabalho de parto e parto. Rev Latino-am Enfermagem. 2006 Mai-Jun 14(3):414-21.

5. Moscovici S. Representações Sociais: Investigações em psicologia social. $6^{\mathrm{a}}$ ed. Petrópolis: Vozes; 2009.

Santos RV, Penna CMM. A educação em saúde como estratégia para o cuidado gestante, puérpera e ao recém-nascido. Texto Contexto Enferm. 2009 Out-Dez (4):652-60. 
7. Progianti JM, Costa RF. Práticas educativas desenvolvidas por enfermeiras: repercussões sobre vivências de mulheres na gestação e no parto. Rev Bras Enferm. 2012 mar-abr 65(2): 257-63.

8. Jodelet D. Representações sociais. In: Jodelet D, organizadora. As representações sociais. Rio de Janeiro: UERJ; 2001.

9. Santos MFS, Almeida LM. Diálogos com a teoria da representação social. $3^{a}$ ed. Recife: Editora Universitária; 2005.

10. Bardin L. Análise de Conteúdo. $5^{\mathrm{a}}$ ed. Lisboa: Edições 70; 2009.

11. McGrath P, Phillips E, Vaughan G. Vaginal birth after Caesarean risk decision-making: Australian findings on the mothers' perspective. Int J Nurs Pract. 2010 Jun 16(3):274-81.

12. Pereira RR, Franco SC, Baldin N. A Dor e o Protagonismo da Mulher na Parturição. Rev. Brasileira de Anestesiologia. 2011 mai-jun 61(3).

13. Figueiredo NSV, Barbosa MCA, Silva TAS, Passarini TM, Lana BN, Barreto J. Fatores culturais determinantes da escolha da via de parto por gestantes. HU Revista 2010 out-dez 36 (4) 296-306.

14. Nilsson C, Bondas T, Lundgren I. Previous birth experience in women with intense fear of childbirth. J Obstet Gynecol Neonatal Nurs. 2010 May-Jun 39(3):298-309.

15. Warren C, Rebecca N, Timothy A, Charity N, Grace M, Jane S, et al. Study protocol for promoting respectful maternity care initiative to assess, measure and design interventions to reduce disrespect and abuse during childbirth in Kenya. BMC Pregnancy and Childbirth. 2013; 13:21.

16. Smith CA, Levett KM, Collins CT, Crowther CA. Relaxation techniques for pain management in labour. Cochrane Database of Systematic Reviews. chrane Database Syst Rev. 2011 Dec 7:(12):009514. 


\section{ciência
púral}

17. Fenwick J, Staff L, Gamble J, Creedy DK, Bayes S. Why do women request caesarean section in a normal, healthy first pregnancy?. Midwifery. 2010 Aug 26(4):394-400.

18. Hodnett ED. Caregiver support for women during childbirth. Cochrane Database Syst Rev. 2000; 2:199.

19. Foucalt M. Microfísica do poder. 25º edição. GRAAL Editora; 2012.

20. Gagnon AJ, Sandall J. Individual or group antenatal education for childbirth or parenthood, or both. Cochrane Database Syst Rev. 2007 Jul 18:(3):002869.

21. Benatti L, Min Marcelo. Parto com amor. $1^{\text {a }}$ ed. Recife: Editora Panda Books; 2011.

22. Bodner K, Wierrani F, Grünberger W, Bodner-Adler B. Influence of the mode of delivery on maternal and neonatal outcomes: a comparison between elective cesarean section and planned vaginal delivery in a low-risk obstetric population. Archives of Gynecology and Obstetrics. 2011 Jun 283(6):1193-98.

23. Cernadas JMC, Mariani G, Pardo A, Aguirre A, Pérez C, Brener P, et al. Nacimiento por cesárea al término en embarazos de bajo riesgo: efectos sobre la morbilidad neonatal. Arch Argent Pediatr. 2010; 108(1):17-23.

24. Busanello J, Kerber NPC, Fernandes GFM, Zacarias CC, Cappellaro J, Silva ME. Humanização do parto e a formação dos profissionais da saúde. Cienc Cuid Saude. 2011 Jan-Mar 10(1):169-175.

lbmetido: $10 / 01 / 2019$

provado: 30/03/2019 